\title{
CEFALEA Y DISMENORREA PRIMARIA EN POBLACIÓN UNIVERSITARIA EN CENTRO SUPERIOR DE ESTUDIOS UNIVERSITARIOS LA SALLE: UN ESTUDIO PILOTO
}

\author{
Isabel Díaz-Martín, MSc ${ }^{1,2,5}$; Gloria Reyes-Bartrina, PT'3,5; Patricia Villaverde-Cano, PT ${ }^{4,5}$; \\ Ignacio Elizagaray-García, MSc $c^{1,2,5}$ \\ 1. Motion in Brains Research Group, Instituto de Neurociencias y Ciencias del Movimiento (INCIMOV), Centro Superior de Estudios Universitarios \\ La Salle, Universidad Autónoma de Madrid, Madrid, España. \\ 2. Centro Superior de Estudios Universitarios La Salle. Universidad Autónoma de Madrid. C/ Ganímedes 11, 28023 Madrid, Spain \\ 3. Centro Residencial La Florida - Sanitas Mayores. C/ Valle de Toranzo, 3, 28023 Madrid, España. \\ 4. Clínica Alfa, C/ Camino de Valladolid, 14, 28250, Torrelodones, Madrid, España. \\ 5. CranioSPain Research Group, Centro Superior de Estudios Universitarios La Salle, Universidad Autónoma de Madrid. C/ Ganímedes 11, 28023 Madrid, Spain
}

Correspondencia:

Ignacio Elizagaray-García, PT, MSc.

Departamento de Fisioterapia. CSEU La Salle. Universidad Autónoma de Madrid. Calle La Salle, no 10, 28023 Madrid, España Teléfono: (+34) 917401980 Fax: (+34) 913571730

E-mail: jeliga@lasallecampus.es

\section{Conflicto de Intereses:}

Los autores declaran no tener ningún conflicto de intereses. Este proyecto no ha sido presentado en ningún evento científico.

Financiación:

Los autores declaran no haber recibido financiación/compensación para el desarrollo de esta investigación.

Comité de Ética:

CSEULS-PI-220/2018

DOI:

https://doi.org/10.37382/jomts.v2i1.21

Recepción del Manuscrito:

5-Febrero-2020

Aceptación del Manuscrito:

6-Mayo-2020

Licensed under:

CC BY-NC-SA 4.0

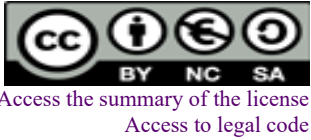

www.jomts.com

\section{RESUMEN}

Objetivo: Describir las características de las mujeres que cursan con cefalea y/o dismenorrea y además, como objetivo secundario, se evaluó la asociación de las variables de dismenorrea y/o impacto de la cefalea con variables psicosociales.

Diseño: Estudio descriptivo observacional comparativo.

Marco: Centro Superior de Estudios Universitarios La Salle (CSEULS).

Participantes: Se reclutó una muestra total de 130 participantes, todas ellas cumpliendo los siguientes criterios de inclusión: ser mujeres en edad fértil comprendida entre los 18 y los 30 años, ser nulíparas, comprender el castellano y haberse sometido a una revisión ginecológica en el último año.

Intervenciones: La recogida de datos consistió en una medición, cada participante rellenó una sola vez los cuestionarios. Los datos recogidos fueron datos demográficos (edad, peso, altura y fecha de la menarquia), la intensidad de la dismenorrea con la escala numérica, el impacto de la cefalea, el grado de ansiedad y depresión, la calidad de vida y el grado de disfunción sexual.

Variables: La intensidad del dolor de la dismenorrea, el impacto de la cefalea, la ansiedad y la depresión, la función sexual y la calidad de vida.

Resultados: Partiendo de una muestra de 130 participantes, 108 presentaron dismenorrea primaria $(83,1 \%)$, el $38 \%$ además cursaban con cefalea. Un $31,5 \%$ presentaba tanto dismenorrea como cefalea. Se dividió la muestra en 4 grupos. El grupo 1 estuvo formado por las participantes que no presentaron dismenorrea ni cefalea $(13,8 \%)$. El grupo 2 lo conformaron los sujetos que solo presentaron dismenorrea $(51,5 \%)$. El grupo 3 fue compuesto por las que solo cursaron con cefalea $(3 \%) \mathrm{y}$, por último, el grupo 4 lo formaron aquellas participantes que padecían tanto dismenorrea como cefalea $(31,5 \%)$.

Conclusión: La dismenorrea tiene una alta prevalencia en el CSEULS. Las mujeres que presentan tanto cefalea como dismenorrea presentan mayores índices de impacto de la cefalea en actividades de la vida diaria, ansiedad y depresión y las participantes que tienen una calidad de vida mayor estadísticamente significativa son las que no tienen ni cefalea ni dismenorrea.

Palabras Clave: Dismenorrea, cefalea, calidad de vida, ansiedad, depresión y disfunción sexual. 


\section{INTRODUCCIÓN}

La dismenorrea es un síndrome doloroso de tipo cólico en la parte inferior del abdomen que acompaña a la menstruación y cursa con espasmos musculares uterinos (Faramarzi y Salmalian, 2014). La localización del dolor suele ser por encima de la sínfisis del pubis y puede verse acompañado de calambres, con irradiación a la zona lumbosacra y muslos. Asimismo, se acompaña normalmente de otros síntomas como cefalea, náuseas y vómitos (López et al., 2001).

La dismenorrea se puede clasificar atendiendo a la intensidad y a su origen. Según su intensidad se puede clasificar en leve, moderada y severa. La dismenorrea leve tiene una duración aproximada de 1 día, la moderada entre 2-3 días y además, se puede acompañar de náuseas, malestar e irritabilidad. Por último, la intensidad severa hace referencia a una duración de 2 a 7 días y no permite hacer las actividades de la vida diaria debido a la intensidad del dolor (López et al., 2001).

Atendiendo al origen de la patología también se puede distinguir entre dismenorrea primaria $\mathrm{y}$ secundaria:

1) La dismenorrea primaria no está asociada a ninguna causa anatomopatológica, ni existe ninguna causa orgánica. Está asociada a los ciclos ovulatorios, debido a las contracciones del miometrio inducidas por las hormonas prostaglandinas, en especial "la prostaglandina PGF2a".

Además, se ha observado que la producción de PGF2a es significativamente superior en las mujeres con dismenorrea. Concretamente, López et al. (2001) señalan que durante las primeras 48 horas de la menstruación es el momento de mayor liberación de estas hormonas, correlacionándose directamente con mayor intensidad de síntomas.

2) La dismenorrea secundaria se asocia a lesiones anatomopatológicas como: endometriosis, tumores, estenosis cervical y anomalías uterinas. Aparece días previos al sangrado menstrual y, del mismo modo que la dismenorrea primaria, el dolor está asociado a una mayor concentración de prostaglandina PGF2a (López et al., 2001).
Este aumento en la concentración de PGH2a que aparece en los sujetos con dismenorrea, tanto primaria como secundaria, también se ha visto en sujetos con migraña común y menstrual (Vardi et al., 1983; Tuca et al., 1989; Antonova, 2013). Estos eicosanoides están involucrados directamente en el proceso nociceptivo y, se ha observado además, que en modelos de dolor inducido por inyecciones de $\mathrm{PGH} 2 \mathrm{a}$ existe una disminución en los umbrales de disparo de las fibras nociceptivas Tipo $\mathrm{C}$, pudiendo ser una de las causas de los múltiples síntomas que presentan estos sujetos (Melzack, 1999).

La sintomatología más frecuente que presentan las jóvenes con dismenorrea está bien establecida en la literatura. Es abundante la literatura que muestra que el síntoma más común que presentan las jóvenes es la presencia de calambres espasmódicos que se inician pocas horas antes de la menstruación y que dura hasta 3 días después (Nagata et al., 2005; Unsal et al., 2010; Rodrigues et al., 2011; Chia et al., 2013; Seven et al., 2014; Shiferaw et al., 2014; Yasir et al., 2014; AlJefout et al., 2015). No obstante, aunque los calambres son el síntoma más común, otros síntomas como el dolor lumbar, mareos, náuseas, vómitos, diarrea y cefalea son frecuentemente descritos por estos pacientes (De Sanctis et al., 2016). Concretamente, se ha encontrado una prevalencia del $18 \%$ de cefalea en un estudio realizado en mujeres de 18 a 45 años con migraña crónica y que padecían dolor menstrual (Spierings y Padamsee, 2015).

Asimismo, la revisión narrativa de De Sanctis et al. (2016) establece conclusiones sobre estudios que abarcan un amplio rango de edades que va desde los 11 hasta los 35 años y no consideran relevante estratificar las conclusiones según diferentes edades.

En esta línea, según Castro y Galleguillos (2009) los sujetos con dismenorrea primaria presentan un mayor número e intensidad de síntomas entre los $20 \mathrm{y}$ 24 años, disminuyendo progresivamente con el avance en el ciclo vital, mientras que Larroy (2015) sugiere que ese mayor número e intensidad de síntomas puede estar relacionado con una mayor concentración hormonal, la cual disminuye a medida que avanzan en el ciclo vital y consecuentemente también los niveles de dismenorrea. En este sentido, parece que el rango de edad en el que la dismenorrea tiene un mayor impacto 
sobre las jóvenes es entre los 20 y 24 años (Castro y Galleguillos, 2009).

En otro orden de cosas, el impacto sobre aspectos psicosociales que acarrea la dismenorrea como por ejemplo; disminución en la calidad de vida, aumento en los niveles de ansiedad y depresión están bien definidos en la literatura (Nagata et al., 2005; Unsal et al., 2010; Rodrigues et al., 2011; Chia et al., 2013; Seven et al., 2014; Shiferaw et al., 2014; Yasir et al., 2014; Al-Jefout et al., 2015; Larroy, 2015; Sahin et al., 2018).

Sin embargo, un único trabajo hasta la fecha valora la presencia de cefalea y su relación con variables psicosociales en población universitaria con una rango de edad de 18-24 años (Yasir et al., 2014). Yasir et al. (2014) llevaron a cabo un estudio descriptivo de una población estudiantil de 356 estudiantes de medicina de la Universidad Islámica de Mohiuddin (Arabia Saudí), mostrando una prevalencia del $56,1 \%$ de jóvenes que presentaban dismenorrea y otro $56 \%$ presentó cefalea dentro del grupo las jóvenes con dismenorrea.

Además, aunque la dismenorrea es frecuente en jóvenes, solo el $25 \%$ de ellas recurre a usar medicación conllevando a ausentarse en los estudios y a interferir en las actividades sociales de su vida diaria. Varios autores, sugieren que las prácticas conductuales de afrontamiento relacionadas con la menstruación y los síntomas relacionados pueden depender de la educación, la actitud, el entorno familiar, la cultura y las creencias de las jóvenes (Harel, 2002; Bekley, 2013).

Atendiendo a la falta de estudios en población universitaria que relacionen la dismenorrea y la cefalea, siendo esta población la que en mayor medida sufre la sintomatología y que además, la gran mayoría de estudios relacionados con este síndrome han sido realizados fuera de España con diferentes costumbres, culturas y creencias; creemos necesario el realizar un estudio observacional comparativo en alumnas universitarias de la Comunidad de Madrid (España) que ayude a continuar aportando conocimiento sobre este problema.

El objetivo principal de este trabajo es describir las características de las jóvenes estudiantes que cursan con cefalea y/o dismenorrea en el Centro Superior de
Estudios Universitarios La Salle y, además, como objetivo secundario se valorará la asociación de las variables de dismenorrea y/o impacto de la cefalea con variables psicosociales.

\section{MÉTODOS}

\section{Diseño de estudio}

Se llevó a cabo un estudio piloto descriptivo observacional comparativo de corte transversal basándonos en las directrices para la realización de estudios observacionales transversales (STROBE) (Unido et al., 2008) en el Centro Superior de estudios universitarios La Salle (CSEULS).

Los evaluadores que realizaron el estudio fueron dos estudiantes de $4^{\circ}$ curso del grado de Fisioterapia de dicha universidad. El proyecto del estudió fue aprobado por el comité de ética del CSEULS con el código CSEULS-PI-220/2018. Todos los procedimientos siguieron las normas éticas de la declaración de Helsinki. La recogida de la muestra se llevó a cabo mediante la captación de las alumnas en el inicio o finalización de las clases en el campus del CSEULS desde enero a abril de 2019.

\section{Participantes}

Para poder participar en este estudio había que cumplir unos criterios de inclusión específicos como ser mujeres en edad fértil comprendida entre los 18 y los 30 años (Habibi et al., 2015), ser nulíparas, comprender el castellano y haberse sometido a una revisión ginecológica en el último año (Ortiz et al., 2015).

Las participantes que quedaron excluidas del estudio fueron aquellas que tomaban anticonceptivos hormonales, si estaban en proceso de embarazo, las que se habían sometido a cirugías abdominales o ginecológicas previas, también quienes padecieran algún tipo de enfermedad infecciosa en el momento de la investigación como hongos o vaginosis, si habían sufrido accidentes de tráfico, tumores, fracturas, enfermedades metabólicas, fibromialgia o dolor de cabeza que no presentara las características de la migraña menstrual producidas por artrosis o hernia discal entre otros (Beltrán-Alacreu et al., 2018). 


\section{Intervenciones}

La recogida de datos consistió en una única medición en la que cada participante rellenó en una única ocasión todos los cuestionarios.

\section{Variables y cuestionarios}

Las variables que se midieron en el estudio fueron: la intensidad del dolor de la dismenorrea, el impacto de la cefalea en actividades de la vida diaria, la ansiedad y la depresión, la disfunción sexual y la calidad de vida.

Intensidad del dolor: Recogida mediante la escala numérica, que es un instrumento de medida en el cual, la paciente debe indicar del 0 al 10 como de intenso es su dolor, siendo 0 nada de dolor y 10 el máximo dolor (Larroy, 2015).

El impacto de la cefalea en actividades de la vida diaria: medida con el cuestionario Headache Impact Test (HIT-6). Es una herramienta para medir el impacto que tienen los dolores de cabeza en la funcionalidad en el trabajo, en la casa, en la escuela y en ámbitos sociales. Está compuesto por 6 preguntas con 4 posibles respuestas: nunca, pocas veces, a veces, muy a menudo y siempre. Si obtuvo una puntuación igual o superior a 60 puntos significa que tiene un impacto muy severo, entre 56 y 59 puntos es impacto importante, entre 50 y 55 cierto impacto y 49 puntos o menos, es poco o ningún impacto (Silva Sieger et al., 2012).

La ansiedad y depresión: medidas con la escala Hospital Anxiety And Depression Scale (HADS). Está compuesta por 14 preguntas, 7 acerca de la ansiedad y otras 7 acerca de la depresión. Se considera que entre 0 y 7 puntos no indica caso, entre 8 y 10 sería un caso dudoso y las puntuaciones superiores a 11 son, probablemente, casos en cada una de las subescalas de ansiedad y depresión (Pérez-López et al., 2012).

La función sexual femenina medida con la escala Female Sexual Function Index (FSFI). Consta de 6 preguntas que miden los niveles de deseo, excitación, lubricación, orgasmo, satisfacción y dolor (PérezLópez et al., 2012).

La calidad de vida medida con el cuestionario SF12 , que refleja la salud física, mental y social. Consta de 12 ítems y se completa en menos de 2 minutos. Las opciones de respuesta forman escalas de tipo Likert que evalúan intensidad o frecuencia. El número de opciones de respuesta oscila entre tres y seis, dependiendo del ítem. Para facilitar la interpretación, estas puntuaciones se estandarizan con los valores de las normas poblacionales, de forma que 50 es la media de la población general. Los valores superiores o inferiores a 50 deben interpretarse como mejores o peores, respectivamente, que la población de referencia. Los ítems son codificados, agregados y transformados en una escala que tiene un recorrido desde 0 (el peor estado de salud para esa dimensión) hasta 100 (el mejor estado de salud) (Vera-Villarroel et al., 2014).

\section{Procedimiento}

Una vez reclutada la muestra, se citó a las participantes para explicarles el procedimiento a seguir en el proyecto de investigación y los cuestionarios a cumplimentar. En esta entrevista se les entregó el consentimiento informado que debían de firmar para poder participar.

Primero les entregamos el consentimiento informado y una vez que nos dieron su consentimiento, pasamos a la entrega de cuestionarios para que lo rellenaran estando nosotras delante por si hubiera alguna duda durante el proceso y nos lo entregaron en mano al finalizar los cuestionarios.

Los datos recogidos fueron datos demográficos (edad, peso, altura y fecha de la menarquia), la intensidad de la dismenorrea con la escala numérica, el impacto de la cefalea, el grado de ansiedad y depresión, la calidad de vida y el grado de función sexual.

La información digital fue guardada y codificada en disco duro bajo custodia al que no tenía acceso ninguna persona ajena al estudio. La información manuscrita fue guardada en un armario cerrado con llave, bajo la custodia del investigador principal y del Centro Superior de Estudios Universitarios La Salle. Si los resultados obtenidos hubiesen tenido alguna influencia relevante sobre la salud de los participantes, éstos serían los primeros en conocer dichos resultados. En el caso que fuese necesario, podría haberse retirado algún paciente del estudio cuando su salud se viese perjudicada.

Los investigadores del estudio eran los únicos que podían tener acceso a los datos de los participantes. 
Además, en caso de utilización de los datos, éstos se utilizaron con el consentimiento de los participantes de manera codificada.

\section{Análisis de resultados}

El análisis de datos se realizó mediante el paquete estadístico SPSS (v.24.0; SPSS, Inc, Chicago, IL). Se empleó la prueba de Kolmogorov-Smirnov $(\mathrm{N}>50$ sujetos) para analizar la normalidad en la distribución de los datos cuantitativos. En este sentido, el valor de $\mathrm{P}$ fue inferior a 0,05 en ciertas variables (edad, peso, menarquia, escala numérica de dismenorrea y FSFI), por lo que se optó por seguir un análisis estadístico con pruebas no paramétricas.

A continuación, se realizó un análisis estadístico descriptivo de los diferentes grupos planteados. Para la formación de grupos se combinó la presencia o no presencia de dismenorrea y/o cefalea. La presentación de los resultados descriptivos se realizó mediante mediana y rango intercuartílico como medidas de tendencia central y dispersión de las variables cuantitativas $\mathrm{y}$, frecuencia absoluta y relativa para las variables categóricas. La diferencia entre los cuatro diferentes grupos formados se evaluó mediante la prueba Kruskal Wallis (Tabla 2). En caso de observarse diferencias entre grupos $(p<0,05)$, se realizó la prueba U de Mann-Whitney comparando los grupos dos a dos con la finalidad de observar entre que grupos existía dicha diferencia $(p<0,05)$ (Tabla 3$)$.

Finalmente, se realizó un análisis de correlación mediante el test Rho de Spearman para probar la existencia de relación entre cada una de las diferentes variables en cada uno de los grupos.

\section{RESULTADOS}

De manera general se obtuvo una muestra de 130 participantes, de las cuales, 108 presentaron dismenorrea primaria $(83,1 \%)$. Dentro de este grupo, el $38 \%$ además cursaban con cefalea. Así pues, del total de la muestra, un $31,5 \%$ presentaba tanto dismenorrea como cefalea (Tabla 1).

Partiendo de estos datos, se dividió la muestra en 4 grupos. El grupo 1 estuvo formado por las participantes que no presentaron dismenorrea ni cefalea. Este grupo agrupó 18 sujetos $(13,8 \%)$. El grupo 2 lo conformaron los sujetos que solo presentaron dismenorrea y agrupó un total de 67 participantes $(51,5 \%)$. El grupo 3 fue compuesto por las que solo cursaron con cefalea y fue formado por 4 personas (3\% de la muestra). El grupo 4 lo formaron aquellas participantes que padecían tanto dismenorrea como cefalea y, agrupó a 41 participantes $(31,5 \%$ de la muestra)

La prueba de Kruskal Wallis (Tabla 2) muestra las comparaciones de las diferencias intergrupo. Posteriormente se realizó un análisis grupo a grupo mediante la prueba U-Mann-Whitney con la intención de albergar entre que grupos se encontraban las diferencias mostradas en la prueba Kruskal Wallis (Tabla 3). La tabla 3 muestra que los participantes del grupo 4 presentaban mayor puntuación en el cuestionario HADS que los sujetos del grupo 1 y 2 $(p=0,000)$. Con respecto al cuestionario HIT- 6 , los resultados muestran que los participantes del grupo 4 obtuvieron mayor puntuación en el cuestionario $(p=0,000)$ que los otros tres grupos. Por último, con relación al cuestionario SF-12 se registró que el grupo 1 obtuvo una puntuación mayor estadísticamente significativa con respecto al grupo $4(p=0,018)$ (Tabla $3)$.

Asimismo, se analizaron las asociaciones de las variables a nivel general. De esta manera, se obtuvo una asociación positiva baja entre la dismenorrea y el cuestionario HADS (Rho $=0,281 ; p=0,003)$, una asociación negativa baja entre la dismenorrea y el cuestionario SF12 (Rho=-0,245; $p=0,011)$, una asociación positiva baja entre los cuestionarios HIT-6 y HADS $(\mathrm{Rho}=0,357 ; \quad p=0,016)$ una asociación negativa moderada entre HIT-6 y el SF-12 $(\mathrm{Rho}=-$ $0,416 ; p=0,004)$ y una asociación negativa moderada entre el HADS y el SF-12 (Rho=-0,670; $p=0,000)$.

Por otro lado, atendiendo a los cuatro diferentes grupos, existió asociación negativa moderada entre el cuestionario HADS y el cuestionario SF-12 en el grupo 1 ( $\mathrm{Rho}=-0,676 ; p=0,002)$. En el grupo 2 se obtuvo una asociación positiva baja entre el cuestionario HADS y la escala numérica de dismenorrea ( $\mathrm{Rho}=0,257 ; p=$ $0,036)$. También se obtuvo una relación negativa moderada entre el cuestionario HADS y el SF12 (Rho=-0,626; $p=0,000)$. En el grupo 4 se registró una asociación positiva baja entre el HIT-6 y el HADS 
Tabla 1. Descripción de la muestra

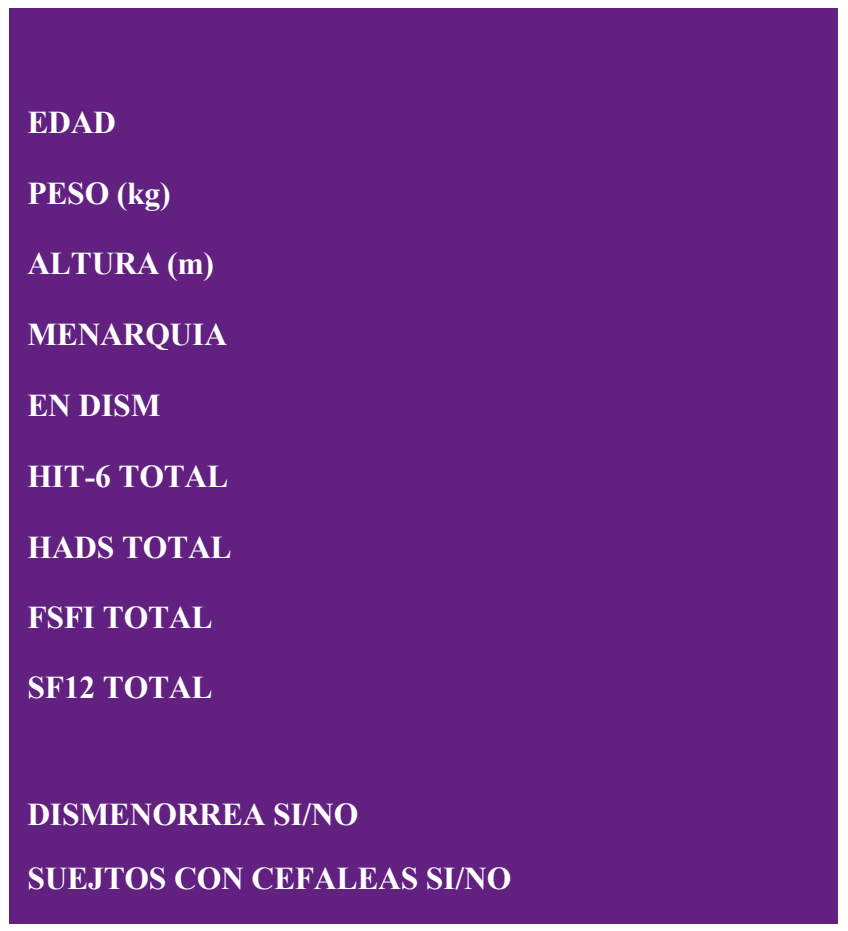

\section{MEDIANA}

21

57

1,64

13

7

61

11

20,50

35,50

$\mathbf{N}$

$108 / 22$

$45 / 85$

\section{RANGO}

(19-22)

$(51-64)$

$(1,64-1,68)$

(12-14)

(5-8)

$(55-65)$

$(8-16)$

$(31-39,25)$

$\%$

$83,1 / 16,9$

$34,6 / 65,4$

Abreviaturas: EN DISM: Escala numérica del dolor. HIT-6 TOTAL: Cuestionario del impacto de dolor de cabeza. HADS TOTAL: Cuestionario de ansiedad y depresión. FSFI TOTAL: Escala de la disfunción sexual femenina. SF12 TOTAL: Cuestionario calidad de vida. $* p<.05$

Tabla 2. Descripción de los grupos y prueba Kruskal-Wallis

\begin{tabular}{|c|c|c|c|c|c|}
\hline & GRUPO 1 & GRUPO 2 & GRUPO 3 & GRUPO 4 & Test Kruskal-Wallis \\
\hline & Mediana (Rango) & Mediana (Rango) & Mediana (Rango) & Mediana (Rango) & Chi-valor ( $p$-valor) \\
\hline EDAD & $19,5(19-22)$ & $21(19-22)$ & $22,5(22-26)$ & $21(19-22)$ & $\mathrm{H}(2)=6,04(\mathrm{p}=0,110)$ \\
\hline PESO (kg) & $58,5(55,75-60,3)$ & $55(50-62)$ & $67,5(57-75)$ & $57(51-66,5)$ & $\mathrm{H}(2)=5,86(\mathrm{p}=0,119)$ \\
\hline ALTURA (m) & $1,64(1,6-1,68)$ & $1,65(1,6-1,69)$ & $1,65(1,57-1,71)$ & $1,63(1,59-1,68)$ & $\mathrm{H}(2)=1,02(\mathrm{p}=0,797)$ \\
\hline MENARQUIA & $13(12,75-14)$ & $12(12-14)$ & $13,5(12-15,75)$ & $12(11,5-14)$ & $\mathrm{H}(2)=5,20(\mathrm{p}=0,152)$ \\
\hline EN DISM & -------- & $7(5-8)$ & ------------ & $7,5(5-8)$ & $\mathrm{H}(2)=1,33(\mathrm{p}=0,248)$ \\
\hline HIT-6 TOTAL & ------- & ---------- & $42,(40,5-51,75)$ & $62(58,5-65,5)$ & $\mathrm{H}(2)=9,11(\mathrm{p}=0,003)^{*}$ \\
\hline HADS TOTAL & $9(6,75-11)$ & $10(8-15)$ & $11(8,25-14,5)$ & $15(10,5-19)$ & $\mathrm{H}(2)=18,61(\mathrm{p}=0,000)^{*}$ \\
\hline FSFI TOTAL & $23(10,5-26)$ & $18(8-25)$ & $12(7,5-22,5)$ & $21(11,5-25)$ & $H(2)=1,46(p=0,691)$ \\
\hline SF12 TOTAL & $40,5(37,7-42,25)$ & $36(32-39)$ & $38(33,5-41,75)$ & $32(29-36)$ & $H(2)=25,60(p=0,000)^{*}$ \\
\hline
\end{tabular}

Abreviaturas: EN DISM: Escala numérica del dolor. HIT-6 TOTAL: Cuestionario del impacto de dolor de cabeza. HADS TOTAL: Cuestionario de ansiedad y depresión. FSFI TOTAL: Escala de la disfunción sexual femenina. SF12 TOTAL: Cuestionario calidad de vida. ${ }^{*} p<.05$ 
Tabla 3. Comparaciones de los diferentes grupos mediante la prueba U-Mann Whitney

\begin{tabular}{|c|c|c|c|c|c|c|}
\hline & Grupo 1 vs grupo 2 & Grupo 1 vs. grupo 3 & Grupo 1 vs. grupo 4 & Grupo 2 vs grupo 3 & Grupo 2 vs. grupo 4 & Grupo 3 vs grupo 4 \\
\hline & U-valor (p-valor) & U-valor (p-valor) & U-valor (p-valor) & U-valor (p-valor) & U-valor (p-valor) & U-valor (p-valor) \\
\hline HIT-6 TOTAL & $\mathrm{U}=594(\mathrm{p}=1,000)$ & $\mathrm{U}=0(\mathrm{p}=0,000)^{*}$ & $\mathrm{U}=0(\mathrm{p}=0,000)^{*}$ & $\mathrm{U}=0(\mathrm{p}=0,000)^{*}$ & $\mathrm{U}=0(\mathrm{p}=0,000)^{*}$ & $\mathrm{U}=6,5(\mathrm{p}=0,003)^{*}$ \\
\hline HADS TOTAL & $\mathrm{U}=452,5(\mathrm{p}=0,104)$ & $\mathrm{U}=23,5(\mathrm{p}=0,285)$ & $\mathrm{U}=138(\mathrm{p}=0,000)^{*}$ & $\mathrm{U}=128(\mathrm{p}=0,881)$ & $\mathrm{U}=843,5(\mathrm{p}=0,001)^{*}$ & $\mathrm{U}=49(\mathrm{p}=0,187)$ \\
\hline SF12 TOTAL & $\mathrm{U}=429,5(\mathrm{p}=0,060)$ & $\mathrm{U}=24(\mathrm{p}=0,305)$ & $\mathrm{U}=226(\mathrm{p}=0,018)^{*}$ & $\mathrm{U}=132(\mathrm{p}=0,960)$ & $\mathrm{U}=1160(\mathrm{p}=0,173)$ & $\mathrm{U}=71(\mathrm{p}=0,658)$ \\
\hline
\end{tabular}

GRUPO 1: dismenorrea no/cefalea no. GRUPO 2: dismenorrea sí/cefalea no. GRUPO 3: dismenorrea no/cefalea sí. GRUPO 4: dismenorrea si/cefalea sí. MENARQUIA: Edad de la primera menstruación. EN DISM: Escala numérica del dolor. HIT-6 TOTAL: Cuestionario del impacto de dolor de cabeza. HADS total: Cuestionario de ansiedad y depresión. FSFI TOTAL: Escala de la disfunción sexual femenina. SF12 TOTAL: Cuestionario calidad de vida. $* p<.05$

(Rho $=0,332 ; p=0,040)$, una asociación negativa baja entre el HIT-6 y el SF12(Rho=-0,332; $p=0,034)$ y una correlación negativa moderada entre el HADS y el $\mathrm{SF} 12\left(\mathrm{Rho}=-0{ }^{\prime} 559 ; p=0,000\right)$.

\section{DISCUSIÓN}

Los resultados obtenidos en este estudio muestran la prevalencia de dismenorrea y cefalea en mujeres universitarias del CSEULS, así como la relación de estas patologías con los factores psicosociales. De manera general, el presente estudio descriptivo muestra una alta prevalencia $(83,1 \%)$ de dismenorrea dentro de la población estudiantil del CSEULS, de las cuales un $38 \%$ presentaron a su vez cefalea y únicamente un $13,8 \%$ no presentaron síntomas. Además, es relevante señalar que las jóvenes que presentaron simultáneamente dismenorrea y cefalea mostraron niveles de calidad de vida significativamente inferiores.

Concretamente, cabe destacar una alta prevalencia de mujeres con dismenorrea en el CSEULS, el 83,1\% de la muestra padecía dicha patología. Este dato concuerda con los resultados de estudios previos sobre la prevalencia de la dismenorrea, donde se reportó que el $61,9 \%$ de las mujeres sufren dismenorrea y que a su vez va disminuyendo esa prevalencia con el avance de la edad debido probablemente a los cambios hormonales que sufre la mujer a lo largo del ciclo vital (Larroy, 2015). El presente estudio muestra que de las mujeres que presentaban dismenorrea, el 38\% además cursaban con cefalea, de este modo, el $31,5 \%$ de la muestra presentaba tanto dismenorrea como cefalea. Por el contrario, el 13,8\% no padecía ninguna de las patologías, siendo un dato relevante, pues indica que poca parte de la población femenina no tiene dolencias de dismenorrea o cefalea asociada a la menstruación. Estos datos concuerdan con el artículo de López et al. (2001) donde se muestra que entre el $48 \%$ y el $90 \%$ de las consultas médicas es debido a la dismenorrea.

En el presente estudio, una pequeña parte de la muestra, el 3\%, engloba a las participantes que tan solo padecían cefalea lo que indica que es poco común que se den estos casos.

En cuanto a los factores psicosociales asociados a la dismenorrea, se vio una importante relación con la ansiedad y la depresión. Las participantes que presentaron dismenorrea y cefalea presentaron niveles más altos de ansiedad y depresión que las que no padecieron dichas patologías. Estos datos concuerdan con estudios realizados anteriormente (Sahin et al., 2018). Estudios previos han estudiado el origen de la relación entre la ansiedad y la depresión con la dismenorrea y cefalea donde sugieren que el origen de esta relación puede ser debida a deficiencias de vitaminas, alteraciones hormonales y desregulación de neurotransmisores (Strine et al., 2005). Así mismo, estos sujetos cursan con mayores dolores de cabeza debido a una disminución de estrógeno durante el ciclo menstrual de la mujer (Faramarzi y Salmalian, 2014).

En relación a la calidad de vida de estas mujeres, se ha comprobado que tienen peor calidad de vida las mujeres que cursan con dismenorrea y cefalea que las que no tienen ninguna patología, lo cual concuerda con anteriores investigaciones (Sahin et al., 2018). Esto puede ser debido a que la presencia de ambos dolores limita las actividades que realizan las participantes. 
Teniendo estos datos en cuenta que el grupo de participantes con mayores niveles de dismenorrea y cefalea presentaron significativamente mayor ansiedad, depresión y una peor calidad de vida que el grupo que solo presento dismenorrea; parece que la cefalea puede ser un factor determinante a la hora de limitar la calidad de vida de los pacientes con dismenorrea.

En este sentido, el ejercicio aeróbico ha mostrado ser una intervención segura y con moderada evidencia para mejorar su calidad de vida de estas personas (La Touche et al., 2020). Además, el ejercicio aeróbico ha mostrado tener efectos profilácticos similares a los de la medicación preventiva de la migraña como es el Topiramato, por lo que podría ser una opción clínica profiláctica a tener en cuenta durante la fase no sintomática del ciclo menstrual en esta población. La mejora en la calidad de vida podría suponer una disminución en el absentismo laboral y una posible disminución en los niveles de ansiedad y depresión. De este modo, se sugiere que futuras líneas de investigación estudien la efectividad del ejercicio aeróbico en sujetos con migraña asociada a la dismenorrea y sus variables psicosociales asociadas.

En relación con la disfunción sexual femenina analizada mediante el cuestionario FSFI, no se han obtenido resultados relevantes en nuestro estudio. Sin embargo, Roa-Meggo (2006) señala que existe asociación entre la dismenorrea y la disfunción sexual. Esta diferencia puede ser debida al enfoque de los estudios, pues el presentado por Roa-Meggo (2006) lo aborda desde una perspectiva más psicológica. Aunque hay estudios sobre la disfunción sexual en relación con otras patologías ginecológicas, no existen muchos estudios acerca de la dismenorrea y la disfunción sexual femenina. Creemos necesario más investigación al respecto.

\section{Limitaciones}

Este estudio piloto transversal presenta de manera implícita varias limitaciones. Primero, el reducido tamaño muestral limita el establecimiento de conclusiones robustas sobre los resultados mostrados. Sin embargo, permitirá a los futuros estudios transversales un cálculo del tamaño muestral más preciso y así ofrecer unos resultados más representativos.
Además, el sesgo de selección implícito a obtener la muestra de un único centro universitario dificulta el principio de representatividad de la muestra y por lo tanto a la extrapolación de los resultados más allá del CSEULS. En esta línea, es posible que variables como el nivel social, económico y cultural puedan determinar la actitud, las creencias y afrontamiento de los síntomas relacionados con la menstruación (Harel, 2002; Bekley, 2013); por lo que los resultados de este trabajo deben ser tomados con cautela. Se sugiere que futuros estudios tengan en cuenta estas variables a la hora de valorar la asociación entre síndromes dolorosos. Asimismo, futuras diseños transversales comparativos sobre población universitaria privada y pública o estudios longitudinales que controlen variables dichas psicosociales podrían ayudar a solventar esta limitación y así, a ampliar el conocimiento sobre la asociación entre la dismenorrea y la cefalea.

Por último, el diseño transversal impide establecer correlaciones causa-efecto relacionadas con las asociaciones encontradas mediante el análisis de correlación realizado (Rho de Spearman), por lo que deberían plantearse futuros diseños longitudinales que controlen estas variables con la intención de subsanar esta limitación.

\section{FRASES DESTACADAS}

$>$ Las mujeres con dismenorrea en el CSEULS ascienden al 83,1\%, el 31,5\% fue afectado por el dolor de cabeza y la dismenorrea.

$>$ Las mujeres afectadas por ambas patologías mostraron un mayor índice de depresión y ansiedad y una menor calidad de vida que las que sólo estaban afectadas por la dismenorrea o ninguna de ellas.

$>$ No se detectó ninguna disfunción sexual femenina en ninguno de los grupos. 


\section{CONCLUSIÓN}

Las mujeres con dismenorrea del CSEULS ascienden a un 83,1\%. Además, las mujeres con síntomas de dismenorrea también se vieron afectadas por el dolor de cabeza en un $38 \%$, y un $31,5 \%$ se vieron afectadas por dismenorrea y dolor de cabeza. Únicamente el $13,8 \%$ de las mujeres encuestadas no presentaron síntomas.

Las mujeres afectadas por ambas patologías mostraron mayor impacto por la cefalea, depresión y ansiedad que las que sólo presentaban o cefalea o dismenorrea de manera aislada.

La calidad de vida se ve a su vez significativamente disminuida en las mujeres con cefalea asociada a la dismenorrea que aquella que no presentaron síntomas. No se encontraron diferencias en cuanto a la disfunción sexual femenina en ninguno de los grupos.

\section{REFERENCIAS}

Al-Jefout M, Seham AF, Jameel H, Randa AQ, Ola AM aitah, Oday AM aitah, Luscombe G. Dysmenorrhea: Prevalence and Impact on Quality of Life among Young Adult Jordanian Females. J Pediatr Adolesc Gynecol. Elsevier Inc.; 2015;28(3):173-85 DOI: http://dx.doi.org/10.1016/j.jpag.2014.07.005.

Antonova M. Prostaglandins and prostaglandin receptor antagonism in migraine. Dan Med J. Danish Medical Association; 2013;60(5) DOI: http://dx.doi.org/10.1186/1129-2377-1-s1-p114.

Bekley K. Psychosocial Aspects of Chronic Pelvic Pain Primary Dysmenorrhea: An Urgent Mandate. Pain Clin Updat. 2013;21(3):1-8.

Beltran-Alacreu H, López-de-Uralde-Villanueva I, Calvo-Lobo C, Fernández-Carnero J, La Touche R. Clinical features of patients with chronic non-specific neck pain per disability level: A novel observational study. Rev Assoc Med Bras. Associacao Medica Brasileira; 2018;64(8):700-9 DOI: http://dx.doi.org/10.1590/18069282.64.08.700.

Castro M, Galleguillos C. Dismenorrea Primaria en Adolescentes: Revisión de la Literatura. Rev LA Soc Chil Obstet Y Ginecol Infant Y LA Adolesc. 2009;16(2):24-36.

Chia CF, Lai JHY, Cheung PK, Kwong LT, Lau FPM, Leung $\mathrm{KH}$, Leung MT, Wong FCH, Ngu SF. Dysmenorrhoea among Hong Kong university students: Prevalence, impact, and management. Hong Kong Med J. 2013;19(3):222-8 DOI: http://dx.doi.org/10.12809/hkmj133807.

Faramarzi M, Salmalian H. Association of Psychologic and Nonpsychologic Factors With Primary Dysmenorrhea. 2014;16(8) DOI: http://dx.doi.org/10.5812/ircmj.16307.

Habibi N, Huang MSL, Gan WY, Zulida R, Safavi SM. Prevalence of Primary Dysmenorrhea and Factors Associated with Its Intensity Among Undergraduate Students: A Cross-Sectional
Study. Pain Manag Nurs. W.B. Saunders; 2015;16(6):855-61 DOI: http://dx.doi.org/10.1016/J.PMN.2015.07.001.

Harel Z. A contemporary approach to dysmenorrhea in adolescents. Pediatr Drugs. 2002;4(12):797-805 DOI: http://dx.doi.org/10.2165/00128072-200204120-00004.

Larroy C. Dismenorrea funcional en la Comunidad Autónoma de Madrid: estudio de la prevalencia en función de la edad Dismenorrea funcional en la Comunidad Autónoma de Madrid: estudio de la prevalencia en función de la edad. 2015;(October).

López LCT, García AT, Estella VB, Serranor MG, Robles MRG. Protocolo de actuación sobre «dismenorrea». 2001;3(3):155-62.

Melzack R. From the gate to the neuromatrix. Pain. Elsevier; 1999;82(SUPPL.1) DOI: http://dx.doi.org/10.1016/S03043959(99)00145-1.

Nagata C, Hirokawa K, Shimizu N, Shimizu H. Associations of menstrual pain with intakes of soy, fat and dietary fiber in Japanese women. Eur J Clin Nutr. 2005;59(1):88-92 DOI: http://dx.doi.org/10.1038/sj.ejcn.1602042.

Ortiz MI, Corte SK, Jaramillo-d1 AP, Murgu1 G. European Journal of Obstetrics \& Gynecology and Reproductive Biology Effect of a physiotherapy program in women with primary dysmenorrhea 2015;194:24-9 http://dx.doi.org/10.1016/j.ejogrb.2015.08.008.

Pérez-López FR, Fernández-Alonso AM, Trabalón-Pastor M, Vara C, Chedraui P. Assessment of sexual function and related factors in mid-aged sexually active Spanish women with the sixitem Female Sex Function Index. Menopause. 2012;19(11):1224 30 DOI: http://dx.doi.org/10.1097/gme.0b013e3182546242.

Roa-Meggo Y. Dismenorrea y actitudes hacia la propia sexualidad en jóvenes universitarias de Lima, Perú. Rev Peru Obstet y Enfermería. 2006;2(2).

Rodrigues AC, Gala S, Neves Â, Pinto C, Meirelles C, Frutuoso C, Vítor ME. [Dysmenorrhea in adolescents and young adults: prevalence, related factors and limitations in daily living]. Acta Med Port. 2011;24 Suppl 2:383-8; quiz 389-92 DOI: http://dx.doi.org/10.20344/amp.1477.

Sahin N, Kasap B, Kirli U, Yeniceri N, Topal Y. Assessment of anxiety-depression levels and perceptions of quality of life in adolescents with dysmenorrhea. Reprod Health. BioMed Central; 2018;15(1):13 DOI: http://dx.doi.org/10.1186/s12978-018-0453-3.

De Sanctis V, Soliman AT, Elsedfy H, Soliman NA, Elalaily R, El Kholy M. Dysmenorrhea in adolescents and young adults: A review in different countries. Acta Biomed. L'Ateneo Parmense; 2016;87(3):233-46.

Seven M, Güvenç G, Akyüz A, Eski F. Evaluating dysmenorrhea in a sample of Turkish nursing students. Pain Manag Nurs. 2014;15(3):664-71 DOI: http://dx.doi.org/10.1016/j.pmn. 2013.07.006

Shiferaw MT eshom., Wubshet M, Tegabu D. Menstrual problems and associated factors among students of Bahir Dar University, Amhara National Regional State, Ethiopia: A crosssectional survey. Pan Afr Med J. 2014;17:246 DOI: http://dx.doi.org/10.11604/pamj.2014.17.246.2230.

Silva Sieger FA, Zarruk Serrano JG, Silva Camargo S, Camacho P, Díaz Silva GA, Díaz Quijano FA, García Gómez RG. 
Influencia de la ansiedad y depresión sobre la discapacidad relacionada con la migraña. Acta Neurológica Colomb. Asociación Colombiana de Neurología; 2012;28(4):194-201.

Spierings ELH, Padamsee A. Menstrual-Cycle and Menstruation Disorders in Episodic vs Chronic Migraine: An Exploratory Study. Pain Med (United States). Blackwell Publishing Inc.; 2015;16(7):1426-32 DOI: http://dx.doi.org/10.1111/pme. 12788.

Strine TW, Chapman DP, Ahluwalia IB. Menstrual-Related Problems and Psychological Distress among Women in the United States. $J$ Women's Heal. 2005;14(4):316-23 DOI: http://dx.doi.org/10.1089/jwh.2005.14.316.

La Touche R, Fernández Pérez JJ, Proy Acosta A, González Campodónico L, Martínez García S, Adraos Juárez D, Serrano García B, Angulo-Díaz-Parreño S, Cuenca-Martínez F, Suso-Martí L, Paris-Alemany A. Is aerobic exercise helpful in patients with migraine? A systematic review and meta-analysis. Scand J Med Sci Sports. Wiley; 2020;sms.13625 DOI: http://dx.doi.org/10.1111/ sms. 13625 .
Tuca JO, Planas JM, Parellada PP. Increase in PGE2 and TXA2 in the saliva of common migraine patients. Action of calcium channel blockers. Headache. 1989;29(8):498-501 DOI: http://dx.doi.org/10.1111/j.1526-4610.1989.hed2908498.x.

Unido R, Unido R, Unido R, Centre NC. Declaración de la Iniciativa STROBE ( Strengthening the para la comunicación de estudios observacionales. 2008;22(2):144-50.

Unsal A, Ayranci U, Tozun M, Arslan G, Calik E. Prevalence of dysmenorrhea and its effect on quality of life among a group of female university students. Ups J Med Sci. 2010;115(2):138-45 DOI: http://dx.doi.org/10.3109/03009730903457218.

Vardi J, Flechter S, Alguati A, Regev I, Ayalon D. Prostaglandin - E 2 Levels in the Saliva of Common Migrainous Women. Headache J Head Face Pain. 1983;23(2):59-61 DOI: http://dx.doi.org/10.1111/j.1526-4610.1983.hed2302059.x.

Vera-Villarroel P, Silva J, Celis-Atenas K, Pavez P. Evaluación del cuestionario SF-12: verificación de la utilidad de la escala salud mental. Rev Med Chil. Sociedad Médica de Santiago; 2014;142(10):1275-83 DOI: http://dx.doi.org/10.4067/S0034-9887 2014001000007.

Yasir S, Kant B, Dar MF aroo. Frequency of dysmenorrhoea, its impact and management strategies adopted by medical students. J Ayub Med Coll Abbottabad. 2014;26(3):349-52. 